\title{
SIMULATION OF THE FLUID-STRUCTURE INTERACTION INVOLVING TWO-PHASE FLOW AND HEXAGONAL STRUCTURES IN A NUCLEAR REACTOR CORE
}

\author{
S. HOUBAR ${ }^{1}$, A. GERSCHENFELD ${ }^{1}$ AND G. ALLAIRE ${ }^{2}$ \\ ${ }^{1}$ DES - Département de Modélisation des Systèmes et Structures (DM2S) \\ CEA, Université Paris-Saclay, F-91191 Gif-sur-Yvette, France \\ sofiane.houbar@cea.fr, antoine.gerschenfeld@cea.fr \\ ${ }^{2}$ Centre de Mathématiques Appliquées, École Polytechnique, Institut Polytechnique de Paris \\ route de Saclay, 91128 Palaiseau Cedex, France \\ gregoire.allaire@polytechnique.fr
}

Key words: Multiphysics, Cavitation, Code Coupling, Fluid-Structure Interaction, ALE, Multi-1D simulation

\begin{abstract}
In order to enhance safety assessments of Sodium Fast Reactors (SFR), some scenarios involving transient Fluid-Structure Interactions (FSI) are investigated using numerical simulation tools. SFRs are indeed quite sensible to mechanical deformations regarding their nuclear power (see [1] for more details). The originality of the scenario presented in the paper is to consider sufficient large mechanical interactions involving a large pressure decrease in the fluid domain. This decrease leads to vaporization of the fluid and then to a different impact on the structures. By means of the open-source software Code_Saturne developed by EDF [2], this scenario is investigated in 2D using a 3-equation model derived from the Navier-Stokes equations while an harmonic model is applied for the mechanical structures. The code coupling is managed using the Newmark algorithm for the mechanical part and a damped fixed point algorithm in order to get a converged coupled FSI problem.
\end{abstract}

\section{INTRODUCTION}

In the framework of the Generation IV International Forum [3] gathering countries involved in the development of the future nuclear reactors, various scenarios are investigated using the numerical simulation tool in order to demonstrate their reliability. Some of those scenarios include complex phenomena by coupling different physics. One of this kind of scenarios concern the impact of mechanical deformations of the core which may have an impact on the safety of the reactor core.

In order to perform the numerical simulation of such phenomena, a correct code coupling framework has to be established in order to avoid numerical instabilities and numerical diffusion / dissipation.

Hence, this paper aims at specifying the algorithms used within the Finite Volume code Code_Saturne (v6.0.1) in order to simulate the fluid-structure interaction involving inter-wrapper flows and hexagonal structures. This case corresponds to a strong coupling due to the thin hydraulic inter-wrapper domain. 


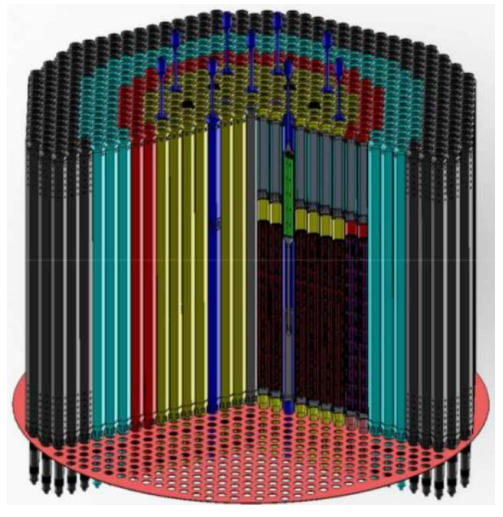

Figure 1: 3D view of a SFR core

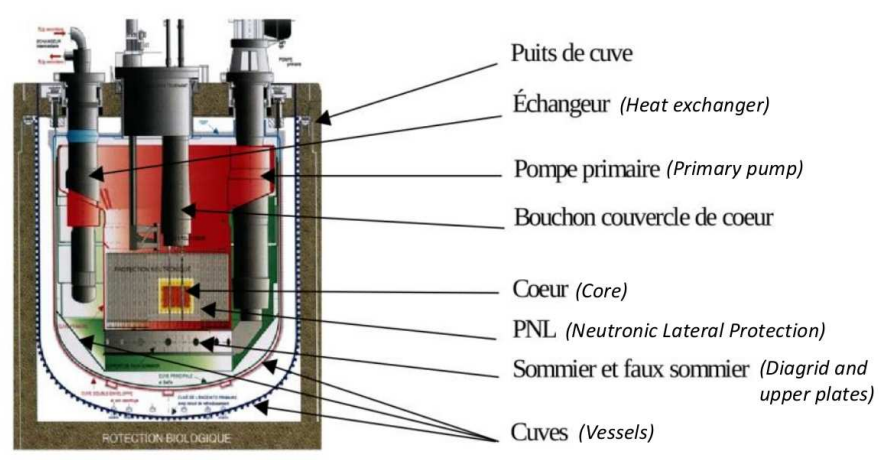

Figure 2: Cross-sectional view of the Phénix reactor core

\section{PRESENTATION OF THE CONTEXT OF THE STUDY}

\subsection{SFR core design}

SFR cores are made of slender hexagonal structures which are highly packed. They are composed with fuel sub-assemblies exchanging the extracted thermal power with circulating liquid sodium (see the mock-up depicted in Figure 1).

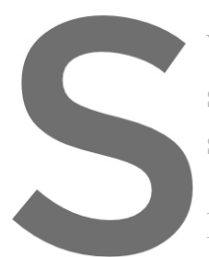

Within the French design, these space between them is filled with st sketch in Figure 2 represents then a If such cores are subjected to mechanical excitatidns due to liquid sodium recirculation in the inter-wrapper region.

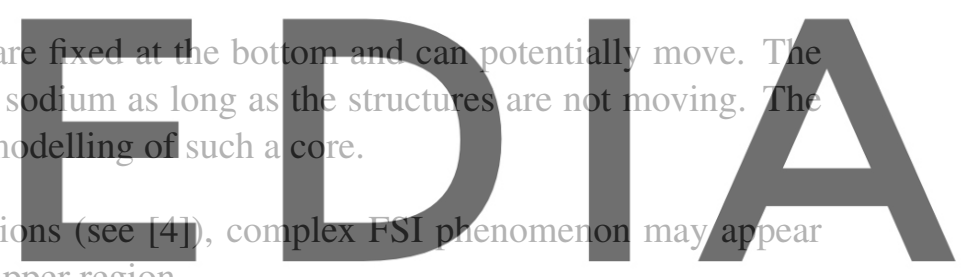

Register for free at https//www.scipedia.com to download the version without the watermark 2.2 Methodology: a reference case

In case of mechanical excitation, the assemblies may move inwards or outwards involving a flow recirculation as depicted in Figure 3. Consequently, the flow recirculation induces a pressure field in the hydraulic region.

\section{HYDRAULIC PHENOMENOLOGY}

\subsection{Presentation of the local scale}

In order to find an law for this field, we consider the simplified case composed with top-bottom plates as depicted in Figure 4. 

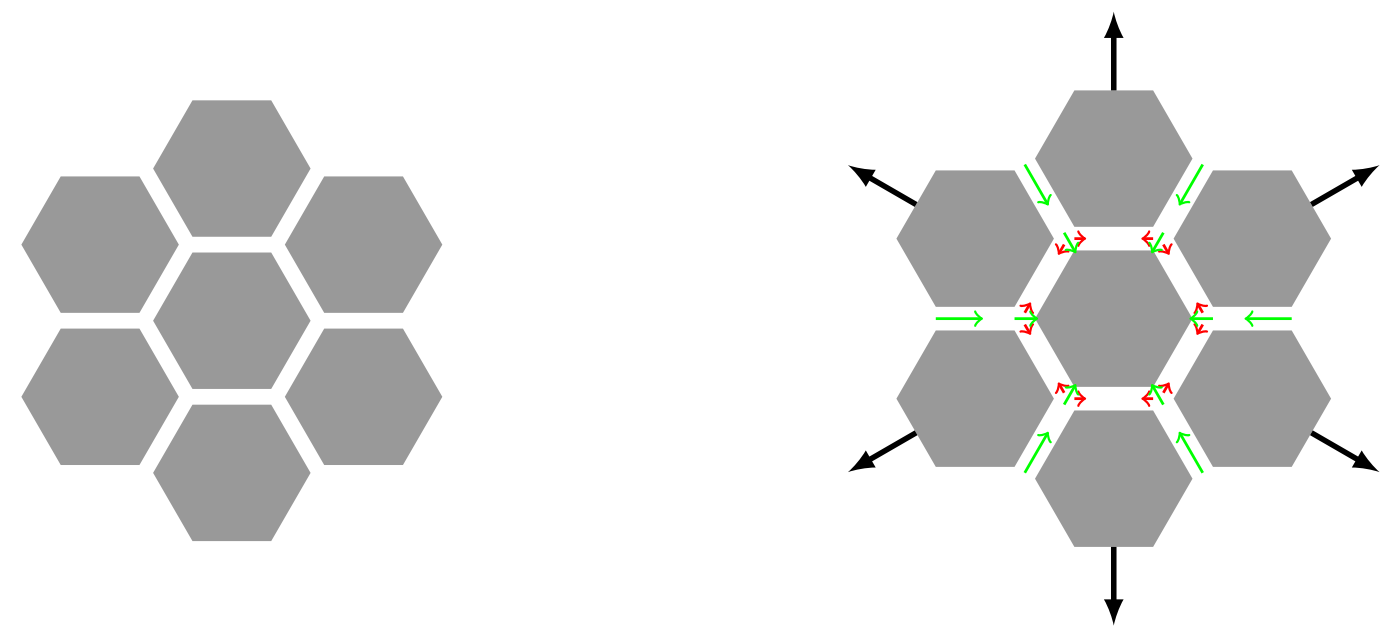

Figure 3: First ring of assemblies in steady state (left) and excited state (right)

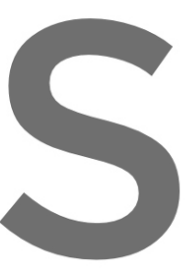

\subsection{Mathematical framework}

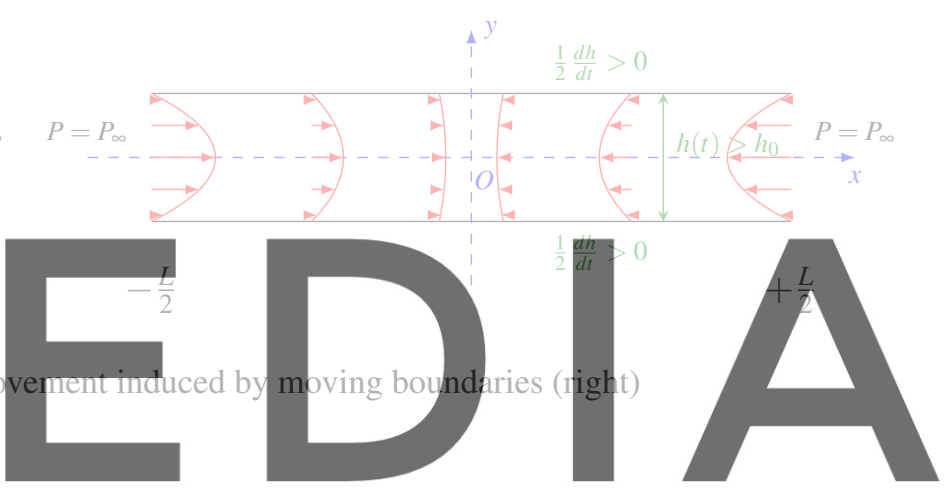

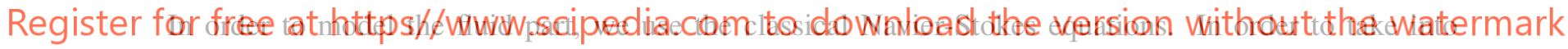
account the movement of the top/bottom boundaries, the ALE (Arbitrary Lagrangian-Euler) technique is used (see for example [5] for the mathematical details of the method). As a consequence, the grid velocity $u_{w}$ is included in Equation (1).

With respect to Figure 4, the following notations are used in order to identify the domain where the Navier-Stokes are solved and where the boundary conditions are set:

$$
\begin{aligned}
\Omega(t) & \stackrel{\text { def }}{=}[-L / 2, L / 2] \times[-h(t) / 2, h(t) / 2] \\
\partial \Omega_{1}(t) & \stackrel{\text { def }}{=}\left\{(x, y) \in \mathbb{R}^{2},|x|=\frac{L}{2}\right\}, t \in \mathbb{R}^{+} \\
\partial \Omega_{2}(t) & \stackrel{\text { def }}{=}\left\{(x, y) \in \mathbb{R}^{2},|y|=\frac{h(t)}{2}\right\}, t \in \mathbb{R}^{+} \\
\partial \Omega_{2}^{-}(t) & =\partial \Omega_{2}(t) \cap\{y<0\} \\
\partial \Omega_{2}^{+}(t) & =\partial \Omega_{2}(t) \cap\{y>0\}
\end{aligned}
$$


According to these notations, the local hydraulic problem is set as follows:

$$
\begin{aligned}
\nabla \cdot \mathbf{u} & =0 \text { in } \Omega(t) \\
\partial_{t} \mathbf{u}+\left[\left(\mathbf{u}-\mathbf{u}_{\mathbf{w}}\right) \cdot \nabla\right] \mathbf{u} & =-\frac{1}{\rho} \nabla P+v \Delta \mathbf{u} \text { in } \Omega(t) \\
\left.P\right|_{\partial \Omega_{1}} & =P_{\infty} \\
\left.\mathbf{u}\right|_{\partial \Omega_{2}^{-}} & =\xi^{-} \\
\left.\mathbf{u}\right|_{\partial \Omega_{2}^{+}} & =\xi^{+}
\end{aligned}
$$

Depending on the movement of the top/bottom boundaries, $\Omega(t)$ changes its volume. It induces a mass conservation depending on time in the volume $\mathcal{V}_{x}(t)$ which is graphically represented in Figure 5.

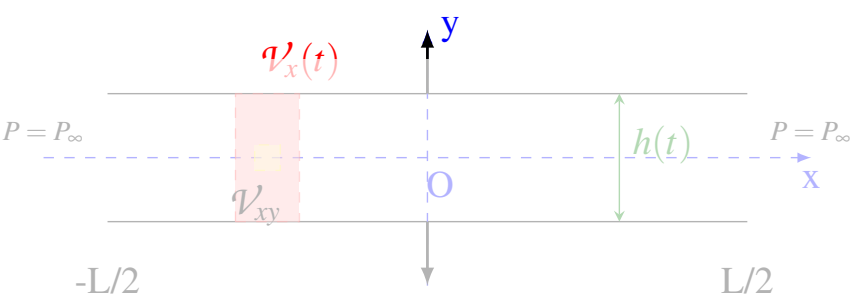

Figure 5: Illustration of the mass conservation law within a hydraulic channel

3.3 Establishing a mutual influence between hydr

Regarding the previous subsection, a movement of th

of the structures, involves a change in the pressure
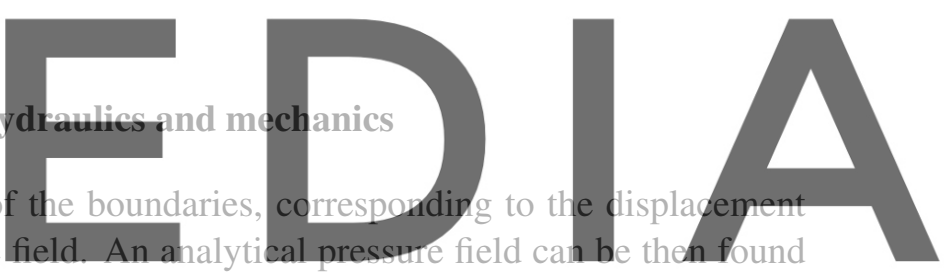

assuming a velocity pattern which respects the mass conservation property in $\mathcal{V}_{x}(t)$ :

Register for free at https//www.scipedia.com to download the version without the watermark

Then, we get an equality for the averaged streamwise velocity (with $u_{x} \stackrel{\text { def }}{=} \mathbf{u}_{\mathbf{x}} \cdot \mathbf{e}_{\mathbf{x}}$ ):

$$
<u_{x}>\stackrel{\text { def }}{=} \frac{1}{h(t)} \int_{h(t)} u_{x} d y=-\frac{h^{\prime}(t)}{h(t)} x
$$

This relationship allows us to find an expression for the averaged pressure over the spanwise direction $y$ designated as $\langle P(x, y, t)\rangle$. The details of the resolution can be found in [6].

$$
\begin{aligned}
<P(x, y, t)>= & P_{\infty}+\frac{\rho}{2 h}\left[\left(h^{\prime \prime}-\frac{h^{\prime 2}}{h}\right)-A_{n} \frac{h^{\prime 2}}{h}\right]\left[x^{2}-\left(\frac{L}{2}\right)^{2}\right]+ \\
& \frac{\alpha \rho v^{\beta}}{2^{2+\beta}(3-\beta)}\left[h^{-3}\left|h^{\prime}\right|^{1-\beta} h^{\prime}\right]\left[|x|^{(3-\beta)}-\left(\frac{L}{2}\right)^{3-\beta}\right]
\end{aligned}
$$


with $A_{n} \in \mathbb{R}^{+}$a factor close to 1 depending on the velocity profile and $(\alpha, \beta)$ a couple of parameters chosen for the friction law represented in the Equation 5:

$$
\int_{h(t)} v \Delta u_{x} d y=-\frac{1}{2 D_{h}} f\left|<u_{x}>\right|<u_{x}>, f=\alpha\left(R e_{x}\right)^{\beta}
$$

with $D_{h}$ depicting the hydraulic diameter and $R e_{x}$ the Reynolds number based on the mean velocity $<u_{x}>$.

This analytical expression applied at the local scale (Figure 4) has shown good agreements with numerical simulation results from different CFD codes (see [6]).

In addition, it can be shown that the pressure law in Eq. 4 can be easily extended to the radial channel such as $I_{0}$ or $I_{2}$ (see Figure 13) by adapting changes in the boundary conditions.

\subsection{Pressure evolution with mechanical forcing}

Using Equation (6), pressure evolution at a local scale depending on the a priori known excitation $h(t)$ can be investigated. We choose to consider an excitation corresponding to a free harmonic system in order to evaluate its effects on the pressure field in the fluid part.
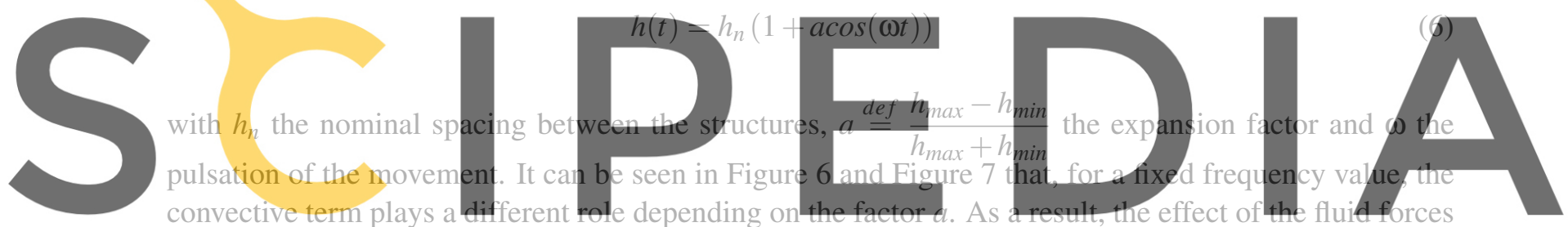

on the structures is different whether the amplitude of the displacement is relatively small or not.

Register for free at https//www.scipedia.com to download the version without the watermark

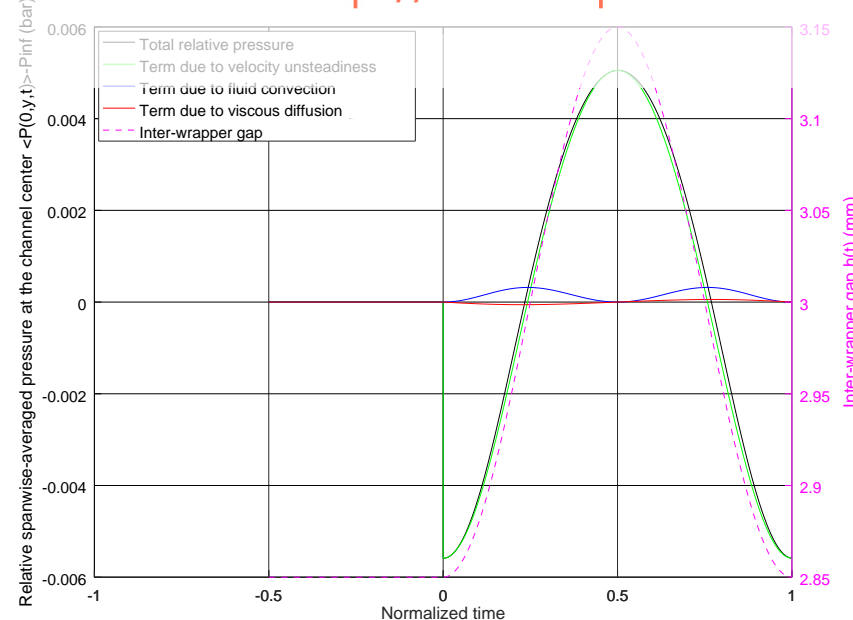

Figure 6: Pressure evolution with $a=0.05$

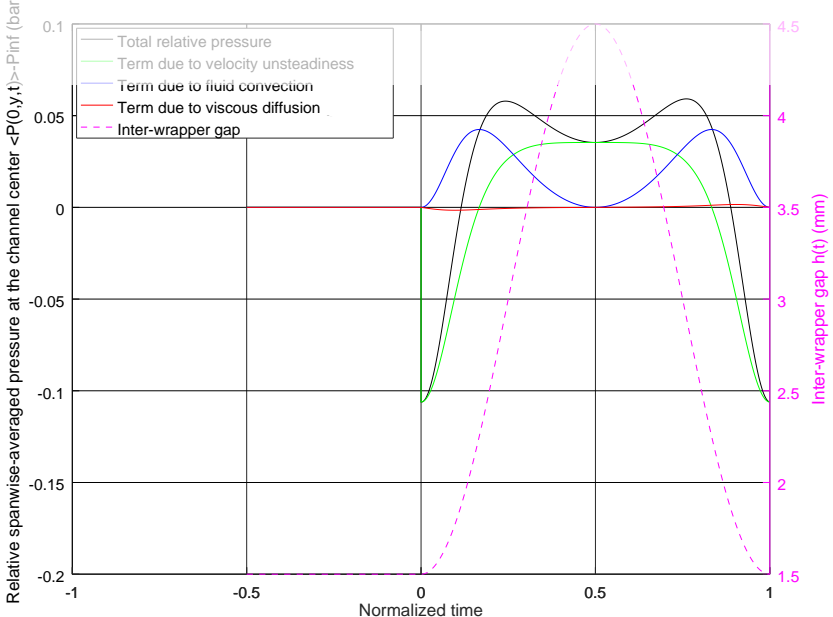

Figure 7: Pressure evolution with $a=0.5$ 


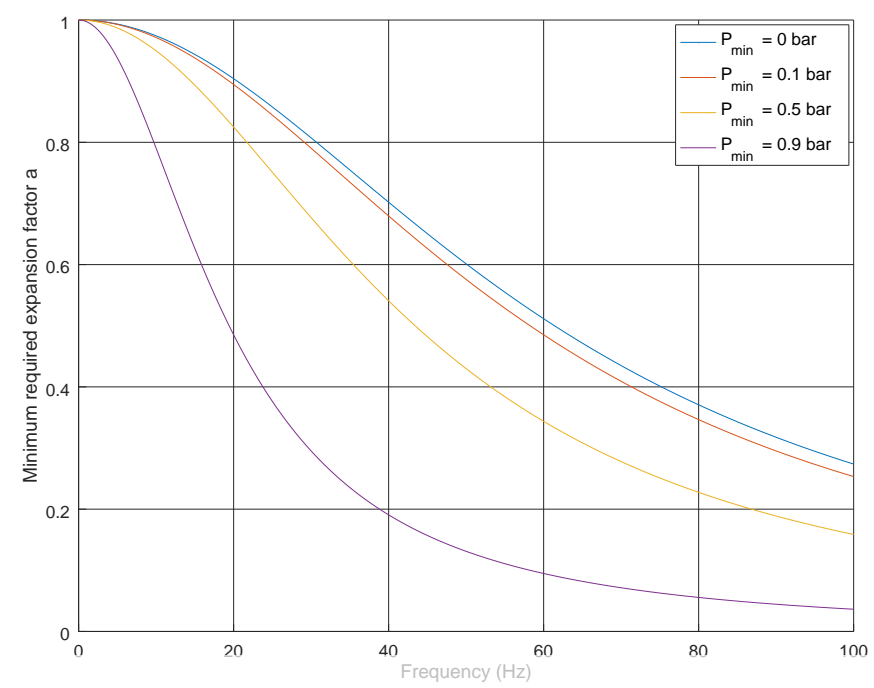

Figure 8: Isovalues of the minimum pressure in the domain $\left(f, a_{\min }\right)$

By including Eq. (6) in (4), we get that the minimum pressure is only due to the second derivative $h^{\prime \prime}$. Figure 8 show the graphical illustration of the conditions on the minimum spacing $h_{\min }$ or frequency of the displacement $f$ in order to reach a minimum pressure.

Moreover, the length of the channel $L$ plays a role in the quanti

3.5 Pressure evolution and Cavitation

When the value of $<P$

in order to take into account the eo-existing vapou
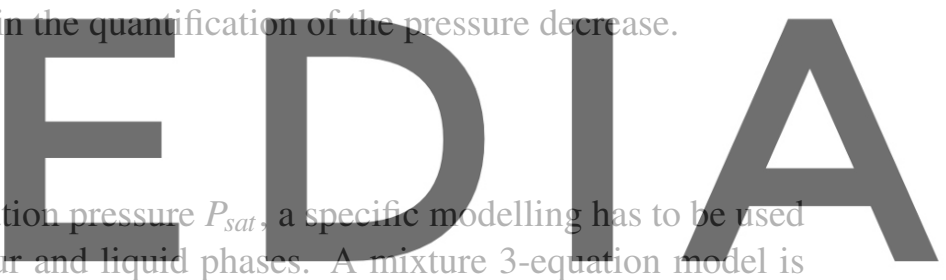

used. In our case, we use the Merkle's model (see [7]):

Register for free at https//www.scipedia.com to download the version, without the watermark

$$
\begin{gathered}
\underbrace{\nabla \cdot \mathbf{u}_{\mathbf{m}}}_{\text {mass baiance }}=\underbrace{\Gamma_{v}}_{\text {exchange ierm }}\left(\frac{1}{\rho_{v}}-\frac{1}{\rho_{l}}\right) \\
\underbrace{\partial_{t} \boldsymbol{\rho}_{m} \mathbf{u}_{\mathbf{m}}}_{\text {unsteadiness }}+\underbrace{\left(\mathbf{u}_{\mathbf{m}}-\mathbf{u}_{\mathbf{w}}\right) \cdot \nabla\left(\boldsymbol{\rho}_{m} \mathbf{u}_{\mathbf{m}}\right)}_{\text {convection }}=\underbrace{-\nabla P}_{\text {driving source }}+\underbrace{\mu \Delta \mathbf{u}_{\mathbf{m}}}_{\text {friction }} \\
\underbrace{\partial_{t} \boldsymbol{\alpha}}_{\text {unsteadiness }}+\underbrace{\left[-\mathbf{u}_{\mathbf{w}} \cdot \nabla(\mathbf{u})+\nabla \cdot(\boldsymbol{\alpha u})\right]}_{\text {transport }}=\frac{\Gamma_{v}}{\rho_{v}}
\end{gathered}
$$

with $u_{m}$ the mixture velocity, $\rho_{m}$ the mixture density, $\alpha$ the void fraction and $\Gamma_{v}=\dot{m}^{+}+\dot{m}^{-}$such as:

$$
\begin{aligned}
& \dot{m}^{+}=-\frac{\rho_{l} \min \left(P-P_{\text {sat }}, 0\right) \alpha(1-\alpha)}{t_{\infty} P_{\text {sat }}} \\
& \dot{m}^{-}=-\frac{\rho_{v} \max \left(P-P_{\text {sat }}, 0\right) \alpha(1-\alpha)}{t_{\infty} P_{\text {sat }}}
\end{aligned}
$$

with $t_{\infty}=1 \mathrm{~ms}$. 
Let us consider the channel case depicted in Figure 4. The application within a refined mesh of such a model compared to the case without cavitation is shown in Figures 9 and 10. The pressure remains constant at $P=P_{\text {sat }}$ where the void fraction field is not negligible.

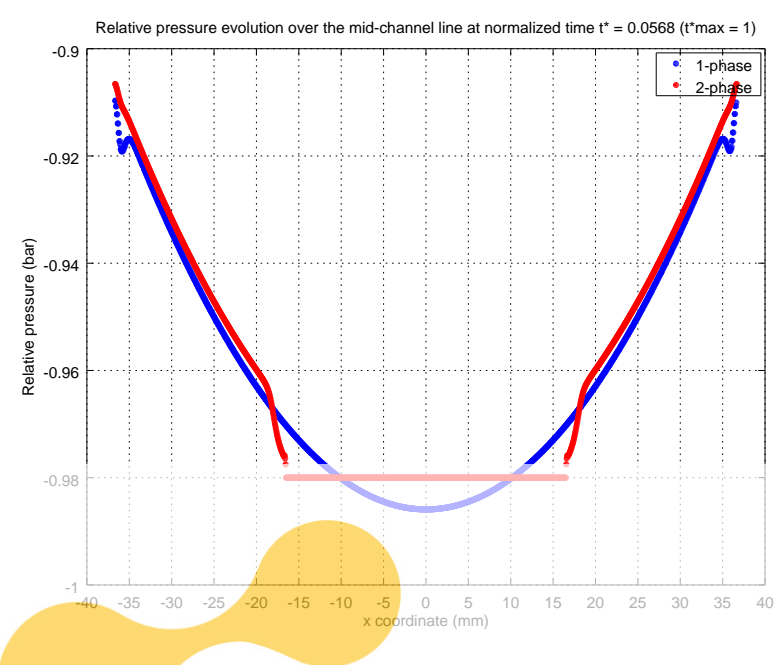

Figure 9: Pressure evolution over $(\mathrm{Ox})$

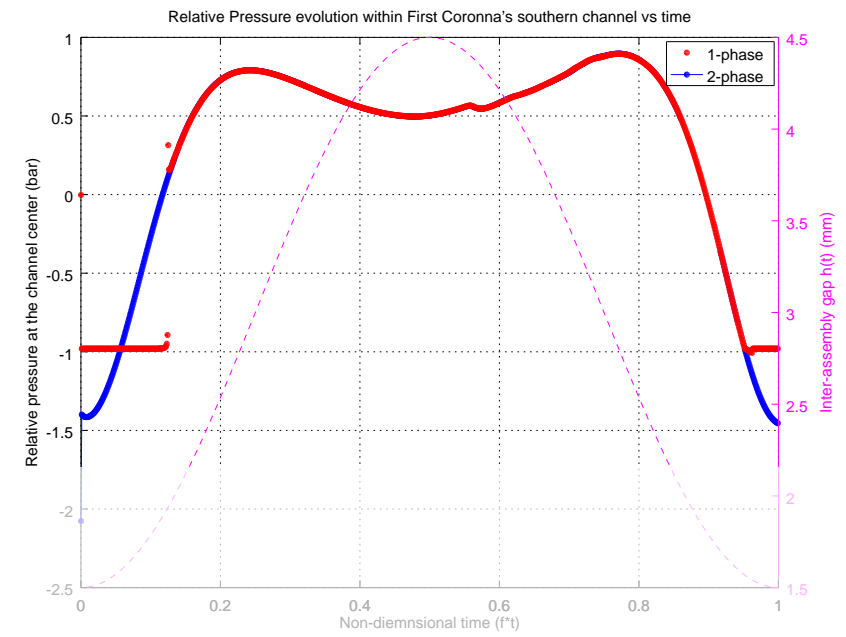

Figure 10: Pressure versus time at the center of the hydraulic channel

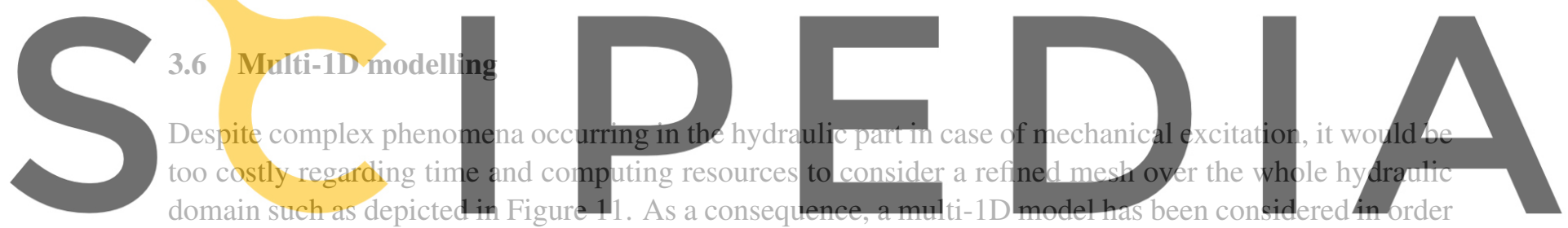

to reduce the cost of the calculations (Figure 12). Within this modelling, only mass conservation, regular

Register fordfrieguat pretps \&/Anawwescipeidradcom to download the version without the watermark

The details of the equations using the multi-1D modelling can be seen in [1].

\section{FLUID-STRUCTURE INTERACTION}

\subsection{FSI modelling}

The general formulation for a damped mass-spring system is considered in order to model the mechanical part as follows (here in tensor notation):

$$
\underline{\underline{M_{i}}} \underline{\ddot{X}_{i}}+\underline{\underline{C_{i}}} \underline{\dot{X}_{i}}+\underline{\underline{K_{i}}} \underline{X_{i}}=\left.\sum_{j} \underline{F}_{F l \rightarrow S t}\right|_{\partial I_{j}}
$$

with the $I_{j}$ the hydraulic channels in connection with the assembly $\mathcal{A}_{i}$ such as depicted in the 3D sketch of Figure 13.

The mechanical part is modelled using a simple harmonic equation in 2D. Then: 


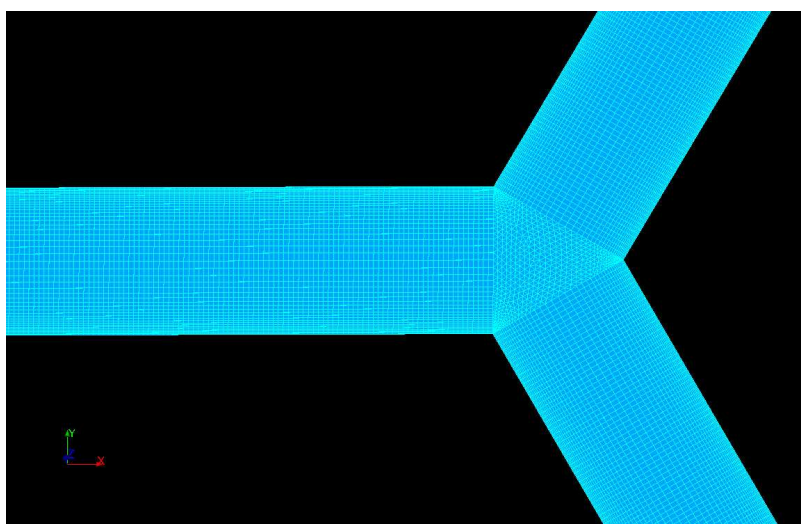

Figure 11: Refined mesh of the hydraulic domain

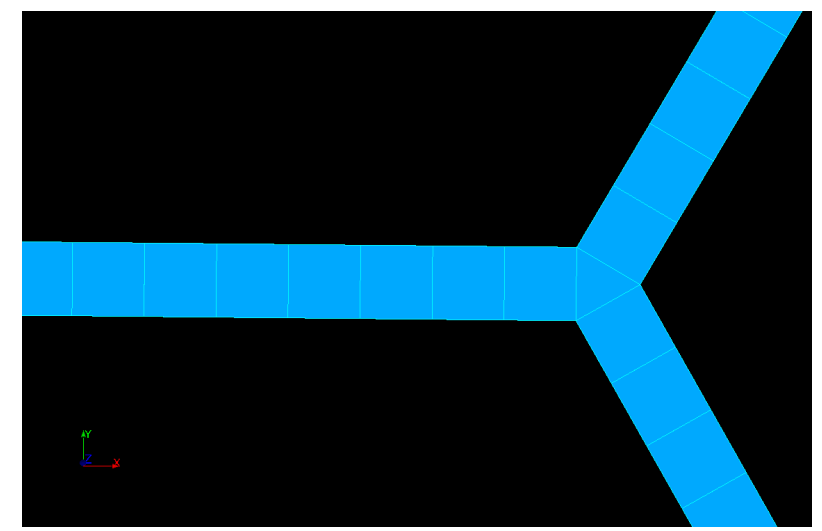

Figure 12: Multi-1D mesh of the hydraulic domain
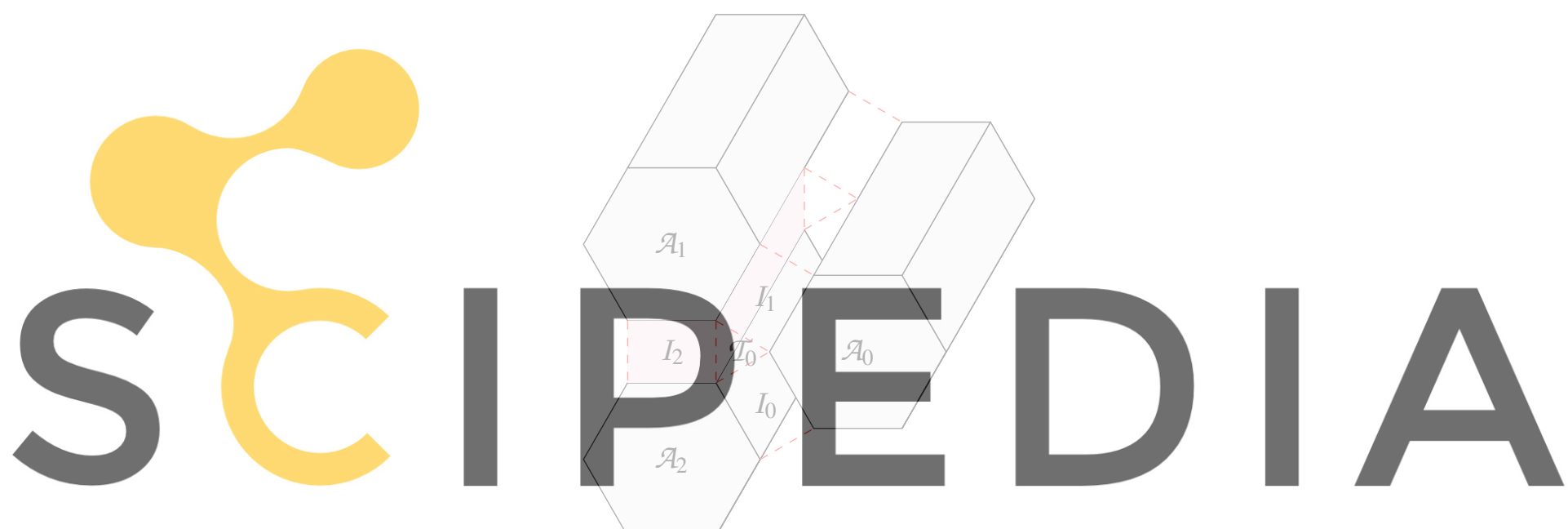
Register for free at https//www.scipedia.com to download the version without the watermark

$$
\begin{aligned}
& \underline{\underline{\underline{M_{i}}}}=\operatorname{diag}\left(m_{i}\right) \\
& \underline{\underline{\underline{\underline{C}_{i}}}}=\operatorname{diag}\left(c_{i}\right) \\
& \underline{\underline{\underline{K_{i}}}}=\operatorname{diag}\left(k_{i}\right) \\
& \underline{\underline{X_{i}}}={ }^{t}\left(x_{i}, y_{i}, 0\right)
\end{aligned}
$$

The fluid forces are assimilated to the pressure forces, neglecting the shear stress effects:

$$
\left.\underline{F}_{F l \rightarrow S t}\right|_{\partial I_{i}}=-\int_{\partial I_{i}} P(x, t) \mathbf{u}_{\mathbf{S t} \rightarrow \mathbf{F l}} d x
$$


Non-linear effects from the fluid part are then introduced. When the displacement is relatively small, only added mass effect from the fluid part is observable. But in case of large displacement, the effects from the fluid part cannot be reduced to added mass effects.

\subsection{Algorithm of the FSI code coupling}

The mechanical and hydraulic parts are solved by two different solvers. Here, the mechanical damping is not considered and then $c_{i}=0$ for any $i$. Taking into account Eqs. (11) and (12), the fluid-structure problem for each assembly number $i$ is discretized as follows:

$$
m_{i} \ddot{x}_{i}^{n+1}+k_{i} x_{i}^{n+1}=\tilde{f}_{i}^{n+1}
$$

The Newmark algorithm is applied for the time-marching using the mean-acceleration scheme:

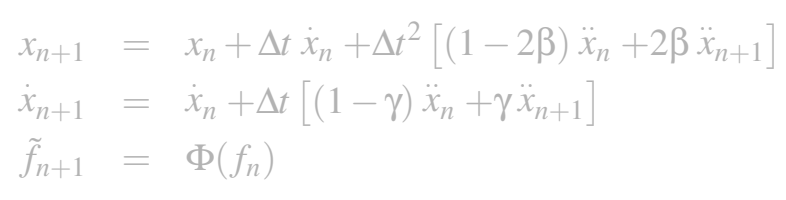

with $\beta=\frac{1}{4}, \gamma=\frac{1}{2} . \tilde{f}_{n+1}$ is an estimation of $f_{n+1}$ at time $n$.

The nature of the transient phenomenon makes this direct code coupling unstable. As a consequence, a damped fixed point algorithm is used in order to ensure the convergence of the coupled problem, with $\lambda$ empirically determined when the ca
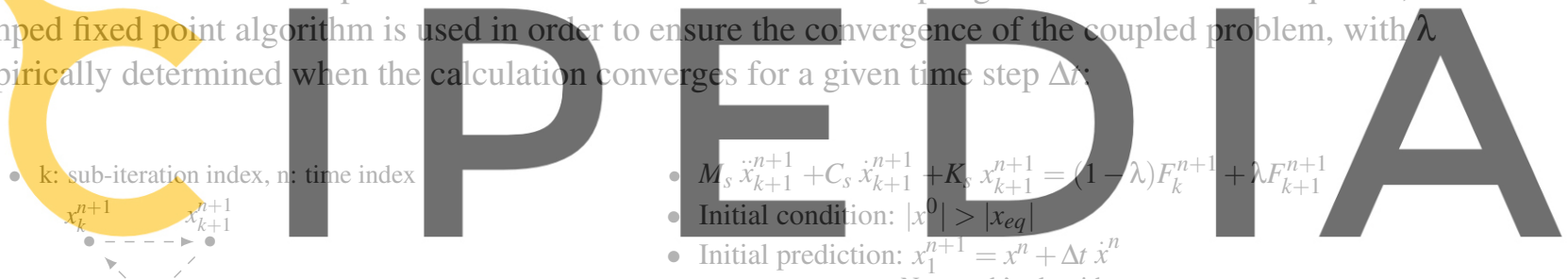

$x_{1}^{n+1}=x^{n}+\Delta t x^{n}$
Newmark's algorithm:

Register for free at hxttps//www.scipedia.com to download the version without the watermark
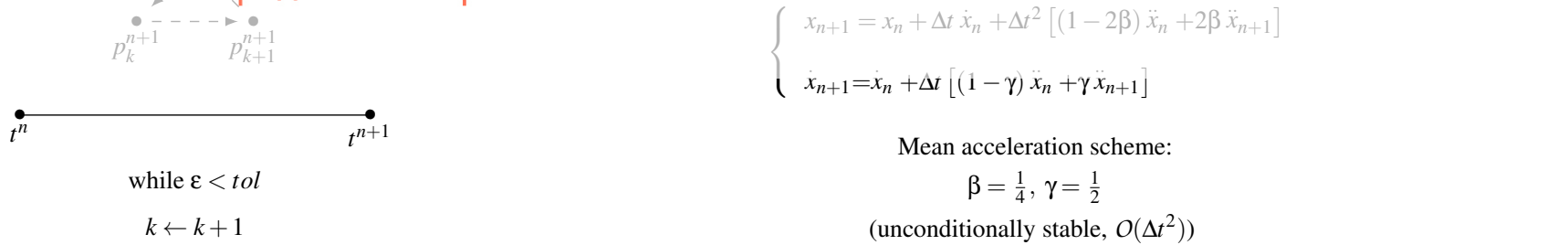

Mean acceleration scheme:

$$
\beta=\frac{1}{4}, \gamma=\frac{1}{2}
$$

(unconditionally stable, $O\left(\Delta t^{2}\right)$ )

\section{SOME RESULTS}

In this section, some computational results of the code coupling algorithm using the multi-1D modelling are presented. The assemblies are initially out of their equilibrium position with $a=3 / 4$.

\subsection{Results of the code coupling within 1 ring}

Here, a configuration with one ring of assemblies is considered. It is a first step in order to see the differences on the displacement field of the assemblies with or without cavitation modelling. The frequency 
is chosen so that the minimum pressure in the domain reaches the value of $P_{s a t}$ in order to have a simulation case with cavitation and FSI code coupling. In this context, Figures 14 and 15 show respectively the effects of cavitation on the pressure fields and void fraction field.

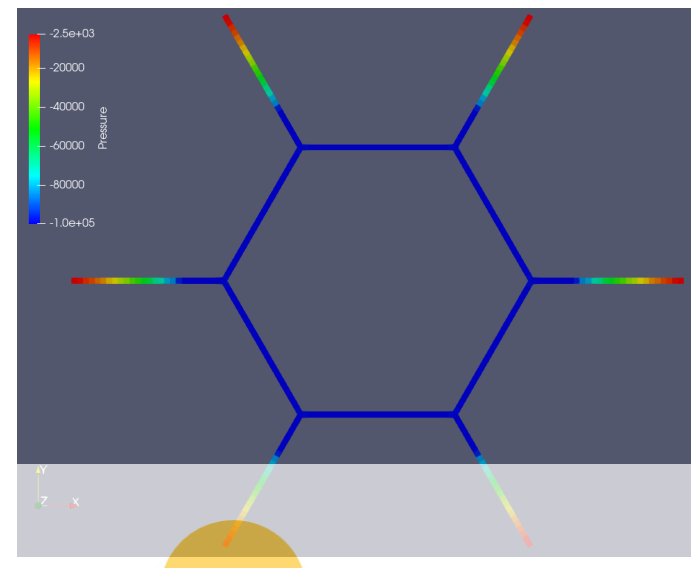

Figure 14: Pressure field

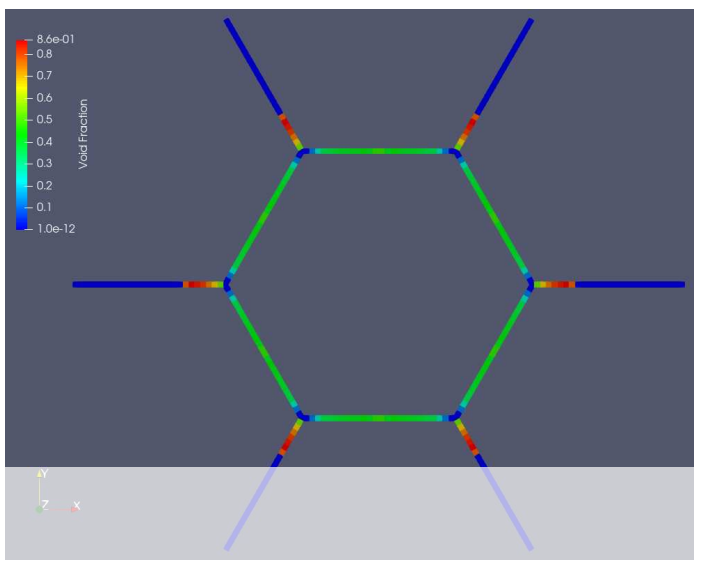

Figure 15: Void fraction field

In comparison with a simulation without cavitation modelling, the pressure can not be lower than the saturation pressure. As a consequence, the restoring forces in the two cases differ from another (see Figure

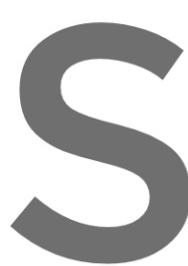

16). Complete re-conder

the change in the variation

taken into account due to ing
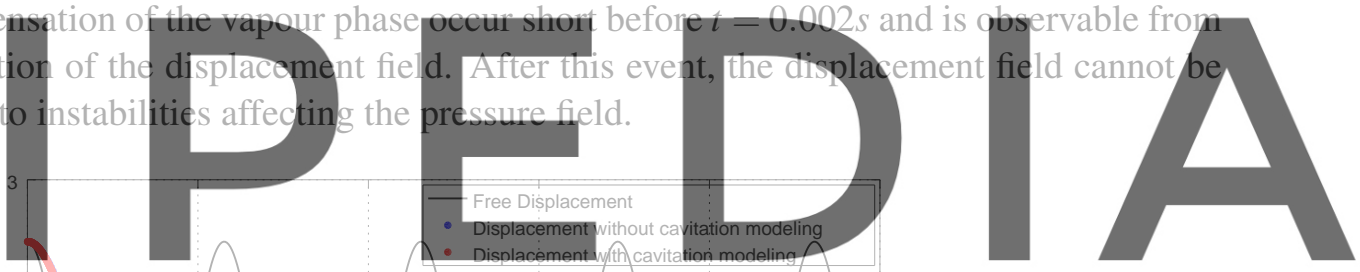

Register for free at https//www.scipedia.com to download the versidn without the watermark

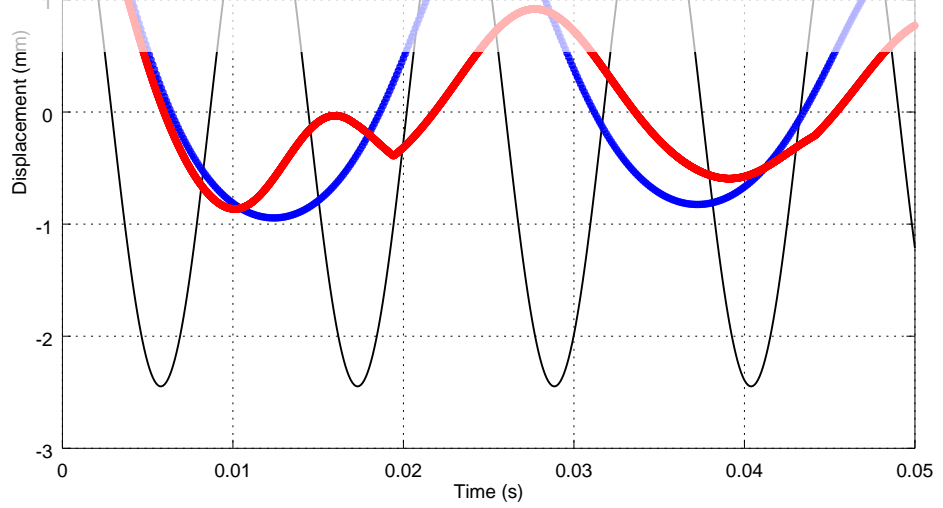

Figure 16: Comparison of the displacements without or with cavitation modelling 


\subsection{Results of the code coupling within 11 rings}

Here, 11 rings are considered in order to represent the real case of a SFR core. In this case, the second eigen-frequency of the assemblies is taken $(f=20 \mathrm{~Hz})$. The pressure fields with or without cavitation modelling are shown in Figures 17 and 18. Vaporization of the fluid occurs next to the center of the core.

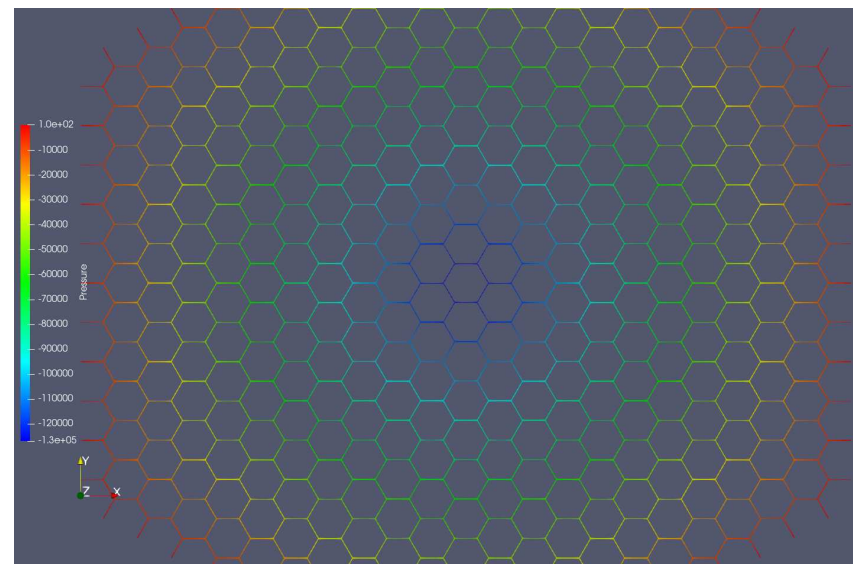

Figure 17: Pressure field with 1-phase modelling

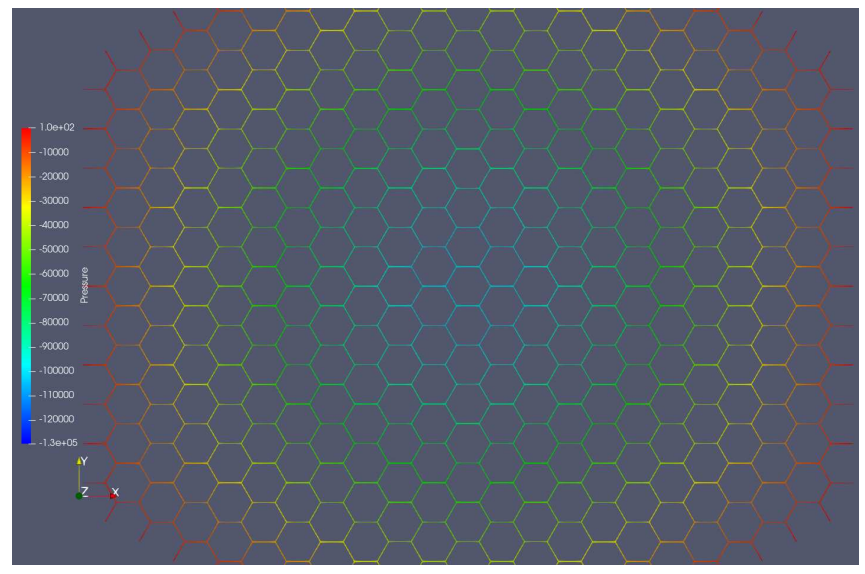

Figure 18: Pressure field with 2-phase modelling

With the same manner as in the previous case with 1 ring of assemblies, the restoring force on the assemblies with cavitation differs from the one without cavitation modelling as depicted in Figure 19. The end of the displacement curve corresponds to the same numerical instability affecting the pressure field which has been previously mentioned in the case with 1 ring.

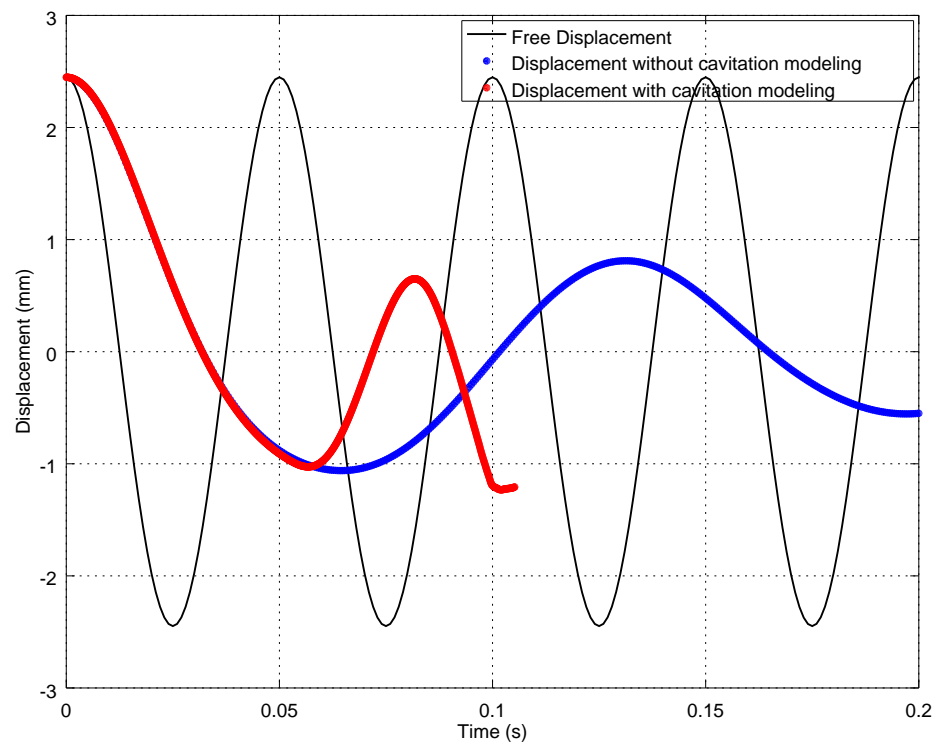

Figure 19: Comparison of the assembly displacements without or with cavitation modelling (first ring) 


\section{CONCLUSIONS}

SFR cores are quite sensible to mechanical deformations and such scenarios may lead to cavitation in the liquid region separating the assemblies. In order to assess such cases, an numerical algorithm coupling two-phase flow and structures within a 2D geometrical representation of a core has been presented.

The algorithm coupling cavitating inter-wrapper liquid sodium and structures makes it possible to predict the impact on the displacement field of the assemblies compared to the case without cavitation modelling.

Nevertheless, the presented numerical algorithm has to be enhanced in order to avoid numerical instabilities when the vapour phases completely disappear.

Moreover, a more detailed analysis concerning the numerical dissipation induced by the FSI numerical algorithm (not presented here) has to be led.

As a perspective, the study has to be extended to $3 \mathrm{D}$ geometries since the movement of the assemblies also induces a flow recirculation at the top of the core. This flow entering into the inter-wrapper region may then have an additional influence on the FSI.

\section{REFERENCES}

[1] Houbar, S., Gerschenfeld, A. and Patricot, C. Study of Cavitation in Liquid Sodium and Simulation of Dynamic Core Deformations. EPJ Web of Conferences (2021) 247:07013.

[2] https://www.code-saturne.org/cms/

[3] https://www.gen-4.org/gif/jcms/c_9261/home

[4] Kepisty, G., Patricot, C., Broc, D. and Campioni, G. SFR mechanical scenarios and neu- tron transport transients with CAST3M code. Annals of Nuclear Energy (2017) 101:226-236

[5] Duarte, F., Gormaz, R. and Natesan, S. Arbitrary Lagrangian-Eulerian method for Navier-Stokes equations with moving boundaries. Computer Methods in Applied Mechanics and Engineering (2004) 193:4819-4836

[6] Houbar, S., Gerschenfeld, A. and Allaire, G. Towards numerical modelling of cavitating interwrapper liquid sodium in SFRs. ECCM-ECCOMAS 2018

[7] Merkle, C. L., Feng, J. Z. and Buelow, P. E. Computational modeling of the dynamics of sheet cavitation Proceedings of the 3rd International Symposium on Cavitation (CAV 98) 307-311, Grenoble, France, April 1998 